\title{
Normas DRIS para cupuaçuzeiro cultivado em monocultivo e em sistemas agroflorestais
}

\author{
Jairo Rafael Machado Dias ${ }^{(1)}$, Daniel Vidal Perez $z^{(2)}$, Lucielio Manoel da Silva ${ }^{(3)}$, \\ Cleigiane de Oliveira Lemos ${ }^{(3)}$ e Paulo Guilherme Salvador Wadt ${ }^{(3)}$
}

\begin{abstract}
(1)Universidade Federal do Acre, Coord. Produção Vegetal, Caixa Postal 500, CEP 69915-900 Rio Branco, AC. E-mail: jairorafaelmdias@hotmail.com (2)Embrapa Solos, Caixa Postal 1.024, CEP 22426-000 Rio de Janeiro, RJ. E-mail: daniel@cpafac.embrapa.br ${ }^{\left({ }^{3}\right)}$ Embrapa Acre, Caixa Postal 321, CEP 69908-970 Rio Branco, AC. E-mail: lucielio@cpafac.embrapa.br, cleigiane@cpafac.embrapa.br, paulo@cpafac.embrapa.br
\end{abstract}

Resumo - O objetivo deste trabalho foi avaliar a performance de normas DRIS específicas ou gerais no diagnóstico do estado nutricional do cupuaçuzeiro (Theobroma grandiflorum), na Amazônia Sul-Ocidental. Amostras foliares de cupuaçu foram coletadas de 153 pomares comerciais, com idade entre 5 e 18 anos, cultivados em monocultivo ou em sistemas agroflorestais. Foram geradas normas DRIS específicas para cada sistema de produção e normas gerais, obtidas para o conjunto da população monitorada. Na obtenção das normas, foi considerada a relação nutricional entre $\mathrm{N}, \mathrm{P}, \mathrm{K}, \mathrm{Ca}, \mathrm{Mg}, \mathrm{Fe}, \mathrm{Cu}, \mathrm{Zn}$ e Mn. A maioria das médias das relações bivariadas entre nutrientes e dos diagnósticos produzidos pelas normas DRIS específicas não diferiu em relação aos produzidos com o uso da norma DRIS genérica. Portanto, a avaliação do estado nutricional de cupuaçuzeiros pode ser realizada com o uso de normas DRIS genéricas, que independem do sistema de cultivo.

Termos para indexação: Theobroma grandiflorum, Amazônia, diagnose foliar, estado nutricional, fruteiras.

\section{DRIS norms for cupuaçu trees cultivated in monocultures and in agroforestry systems}

\begin{abstract}
The objective of this work was to evaluate the performance of specific or generic DRIS norms regarding diagnosis of the nutritional state of cupuaçu (Theobroma grandiflorum) orchards, in Southwestern Amazonia. Cupuaçu leaf samples were collected from 153 commercial orchards with ages ranging from 5 to 18 years, cultivated either in monoculture or in agroforestry systems. Specific DRIS norms, obtained for each production system, and generic norms, for the entire population, were generated. DRIS norms were obtained considering the nutritional relationship between $\mathrm{N}, \mathrm{P}, \mathrm{K}, \mathrm{Ca}, \mathrm{Mg}, \mathrm{Fe}, \mathrm{Cu}, \mathrm{Zn}$ and $\mathrm{Mn}$. The majority of bivariate relation means between nutrients and diagnostics obtained from the specific DRIS norms did not differ in comparison to the ones obtained when the generic DRIS norm was used. The evaluation of the nutritional state of cupuaçu orchards can be done using generic DRIS norms, which do not take into account the production system.
\end{abstract}

Index terms: Theobroma grandiflorum, Amazonia, foliar diagnosis, nutritional state, fruit trees.

\section{Introdução}

A avaliação do estado nutricional de pomares pode ser realizada por meio da diagnose foliar (Veloso et al., 2002), em razão da correspondência que normalmente existe entre a concentração de nutrientes nas folhas e o crescimento, o desenvolvimento e a produtividade das plantas.

Teoricamente, o prognóstico de adubações em fruteiras pode ser estimado pela quantificação da capacidade do solo em suprir os nutrientes necessários por meio da interpretação de análise de solo e pela avaliação do estado nutricional das plantas realizada por interpretação da análise foliar. Contudo, essas interpretações dependem de ensaios de calibração realizados em vários anos e locais (Canterella, 2007), o que torna o processo complexo e oneroso.

A interpretação da análise foliar é realizada pela comparação da concentração dos nutrientes nas folhas e de seus valores (níveis críticos, NC) ou faixas de valores (faixas de suficiência, FS) críticos, acima dos quais não se espera acréscimo na produtividade das plantas pelo aumento na disponibilidade dos nutrientes. 
Os métodos NC ou FS têm sido aplicados com sucesso em várias culturas, anuais ou perenes (Prado et al., 2008). No entanto, a eficiência desses métodos na diagnose nutricional das plantas é influenciada por diversos fatores que não estão diretamente relacionados com a disponibilidade de nutrientes, como cultivar, luminosidade, temperatura, regime hídrico, doenças entre outros, mas que afetam o acúmulo de matéria seca pelas plantas (Jarrel \& Beverly, 1981). Assim, tanto NC como FS somente representariam adequadamente o estado nutricional das culturas com relação a um determinado nutriente em condições ambientais e de manejo semelhantes àquelas nas quais esses parâmetros foram determinados.

O sistema integrado de diagnose e recomendação (DRIS) utiliza, no processo de análise, relações bivariadas entre os nutrientes, o que minimiza o efeito proporcionado pela taxa de acumulação de biomassa (Wadt \& Novais, 1999) e possibilita que suas normas sejam aplicáveis a situações diversas das que foram utilizadas em sua elaboração (Walworth \& Sumner, 1987).

Muitos trabalhos têm discutido as condições ideais para a obtenção das normas DRIS, com resultados controversos. Existem trabalhos que apontam para a possibilidade de obtenção de normas a partir de dados calibrados localmente, como também conclusões que sugerem a utilização de normas DRIS genéricas ou universais. Silva et al. (2005), ao avaliar a universalidade de aplicação das normas DRIS, recomendaram a utilização de normas específicas. Da mesma forma, Rocha et al. (2007) destacaram a importância de obtenção de normas regionais específicas para avaliação do estado nutricional do milho em diferentes condições de cultivo. Galíndez et al. (2009) constataram que é possível a obtenção de normas DRIS mesmo com uma amostragem restrita, com uso de pequeno número de amostras foliares, desde que isso seja feito em condições ambientais homogêneas. Entretanto, Reis Junior (2002) destacou a possibilidade de utilização de normas universais, o que estaria de acordo com a proposição original do método (Beaufils, 1973; Walworth \& Sumner, 1987), quando as condições de cultivo das subpopulações de referência e de amostra são semelhantes.

Para o cupuaçuzeiro, além de não haver normas DRIS disponíveis, não se tem indicação de qual conjunto de normas, universais ou específicas, seria mais adequado, considerando-se especialmente que a cultura é cultivada na Amazônia, em uma grande diversidade de condições, seja quanto à densidade de plantio, presença ou não em consórcios florestais e aos sistemas de manejo (Alfaia \& Ayres, 2004; Ayres \& Alfaia, 2007).

O objetivo deste trabalho foi avaliar a performance de normas DRIS específicas ou gerais no diagnóstico do estado nutricional do cupuaçuzeiro, na Amazônia.

\section{Material e Métodos}

Para a geração das normas DRIS, foram monitorados 153 pomares comerciais de cupuaçuzeiro (Theobroma grandiflorum) - 42 cultivados em monocultivo e 111 cultivados em sistemas agroflorestais (SAFs) - localizados na área de influência do distrito de Nova Califórnia, extremo-oeste do Município de Porto Velho, Rondônia, em um quadrículo contido entre $9^{\circ} 24^{\prime} 45^{\prime \prime}-9^{\circ} 54^{\prime} 54^{\prime \prime S}$ e $65^{\circ} 27^{\prime \prime} 28^{\prime \prime}-65^{\circ} 51^{\prime} 52^{\prime \prime} \mathrm{W}$. A idade dos pomares variou de 5 a 18 anos.

A amostragem das folhas foi realizada no período de julho a setembro de 2008. Coletaram-se, aleatoriamente, duas folhas por planta, nas faces de exposição norte e sul. As amostras foliares consistiram do limbo e pecíolo de folhas completamente expandidas, na posição do terceiro lançamento, a partir do ápice do ramo, localizado na altura média da árvore, no total de 30 folhas por pomar (Costa, 2006).

As análises químicas das amostras foliares foram realizadas por meio de digestão nitroperclórica e sulfúrica. Após a digestão nitroperclórica, as folhas foram analisadas quanto à concentração de $\mathrm{Ca}, \mathrm{Mg}$, $\mathrm{Mn}, \mathrm{Fe}, \mathrm{Zn}, \mathrm{Cu}$ (espectrometria de plasma ICP-OES), $\mathrm{K}$ (fotometria de chama) e de $\mathrm{P}$ (espectrofotometria molecular). O nitrogênio total foi obtido após digestão sulfúrica e destilação por Kjehdahl (Carmo et al., 2000).

No momento da amostragem, cada pomar foi previamente classificado quanto à sua sanidade e qualidade fitotécnica, levando em consideração aspectos fitossanitários, culturais e de solo. Com relação aos aspectos fitossanitários, avaliou-se a infestação da vassoura-de-bruxa (Crinipellis perniciosa) e da broca-do-fruto (Conotrachelus humeropictus), que são os problemas que mais afetam a produtividade na região (Lopes \& Silva, 1998). Quanto ao manejo cultural, avaliaram-se a utilização de podas, permanência de frutos estragados na área de cultivo 
e limpeza da área. Na avaliação do manejo do solo, levaram-se em consideração a presença de adubação orgânica, a cobertura do solo e o cultivo em nível. Para as características estado fitossanitário, manejo cultural e do solo foram atribuídos conceitos: 1, ruim; 2, regular; e 3, bom. Para o estabelecimento das normas, o banco de dados foi dividido em pomares não sadios, com potencial de baixa produtividade (PBP), e pomares sadios, com potencial de alta produtividade (PAP). O critério utilizado para definição das classes foi a soma dos referidos conceitos, em que: $3 \leq \mathrm{PBP} \leq 7$ e $8 \leq \mathrm{PAP} \leq 9$.

Foram determinadas normas DRIS específicas para cupuaçuzeiros cultivados em monocultivo (CCM) ou em sistemas agroflorestais (CCS). Além dessas, determinou-se a norma geral, para cupuaçuzeiros cultivados em qualquer um dos sistemas de produção (CCMS).

Para o cálculo dos índices nutricionais (Inut) e normas DRIS, utilizou-se o software DRIS para cupuaçu (DRIS, 2010), com uso da fórmula de Jones (1981), tendo sido incluídas todas as relações bivariadas diretas e inversas entre os nutrientes avaliados, sem a utilização da transformação logarítmica. $\mathrm{O}$ índice de balanço nutricional médio (IBNm) foi calculado pela média aritmética do somatório dos valores absolutos dos Inut gerados para cada amostra.

Para o cálculo das normas, foram determinados média, variância e número de observações para cada relação entre dois nutrientes, em sua forma direta ou inversa. Para estabelecimento das normas específicas, foram utilizados os pomares sadios, com potencial de alta produtividade de acordo com os critérios fitotécnicos e fitossanitários adotados, sob cultivo solteiro (14 pomares) ou cultivados em SAFs (34 pomares). Para o estabelecimento da norma geral, foram utilizados esses 48 pomares sadios, independentemente do sistema de cultivo. O tamanho das populações, embora pequeno, pode ser considerado suficiente para a geração de normas DRIS, se elas forem constituídas por pomares sadios (Mourão Filho et al., 2002).

A interpretação dos índices DRIS foi realizada pelo método do potencial de resposta à adubação (PRA), a partir de cinco classes (Wadt, 2005): PRA nulo $(\mathrm{Z})=\mid$ Inut $\mid<$ IBNm; PRA positivo ou nulo $(\mathrm{PZ})=\mid$ Inut $\mid>\mathrm{IBNm}$, desde que Inut $<0$; PRA positivo $(\mathrm{P})=\mid$ Inut $\mid>$ IBNm, desde que o Inut avaliado seja o menor índice DRIS entre os demais nutrientes; PRA negativo ou nulo $(\mathrm{NZ})=\mid$ Inut $\mid>\operatorname{IBNm}$, desde que Inut $>0$; e PRA negativo $(\mathrm{N})=\mid$ Inut $\mid>$ IBNm, desde que o Inut avaliado seja o maior índice DRIS entre os demais nutrientes. Tanto o IBNm quanto o Inut são adimensionais.

O PRA nulo é obtido quando o nutriente está em uma faixa de relativo equilíbrio nutricional, o que é refletido por seu valor em módulo inferior ao do IBNm. O PRA positivo ou nulo (PZ) ocorre quando o nutriente encontra-se em deficiência (índice negativo e superior, em módulo, ao do IBNm), mas sem que o nutriente apresente o menor índice DRIS, que é a condição para ocorrência do PRA positivo (P). Essa distinção entre PZ e P ocorre em razão da probabilidade de resposta, que aumenta quando índice é negativo e maior que o do IBNm, ser maior ainda quando o nutriente é o mais negativo. O PRA negativo é interpretado como excesso do nutriente, com índice DRIS positivo (superior ao do IBNm) e com maior valor entre os demais nutrientes. Nesse caso, é mais provável a ocorrência de uma resposta produtiva negativa do que positiva, pela adição do nutriente (Wadt, 2005).

A variância dos teores e das relações bivariadas entre nutrientes foram comparadas nas populações de referência CCM, CCS e CCMS pelo teste $\mathrm{F}$, a $5 \%$ de probabilidade. Mesmo quando o teste F indicou ausência de diferença significativa entre as variâncias, utilizouse o teste $t$ de Student, também a 5\% de probabilidade, para a comparação das médias. Isso porque a ausência de significância no teste $\mathrm{F}$ indica apenas que os teores ou relações foram obtidos em ambientes relativamente homogêneos, e não que é impossível a ocorrência de médias distintas nesses ambientes, já que suas variâncias, no método DRIS, advêm de um grande número de fatores (condições não controladas), e não apenas da disponibilidade dos nutrientes.

A correlação entre os IBNm obtidos nos diferentes pomares, com o uso das diferentes normas DRIS, foi determinada pelo teste de correlação de Pearson, a 1\% de probabilidade.

Foram identificados, em cada um dos pomares, os nutrientes que estavam insuficientes (PRA positivo e PRA positivo ou nulo), em equilíbrio (PRA nulo) e em excesso (PRA negativo e PRA negativo ou nulo), segundo o diagnóstico produzido por cada uma das normas DRIS. Em seguida, avaliou-se o grau de concordância entre os diagnósticos obtidos pelo uso 
das diferentes normas. Se, para um dado nutriente, o diagnóstico (insuficiência, equilíbrio ou excesso) foi o mesmo entre duas normas distintas, ele foi considerado concordante. Se o diagnóstico foi distinto, ele foi considerado não concordante. Computou-se, então, a percentagem de diagnósticos concordantes, para o total de pomares avaliados.

Finalmente, a frequência com que cada nutriente foi identificado como tendo respostas do tipo PRA nulo, PRA positivo, PRA positivo ou nulo, PRA negativo e PRA negativo ou nulo, dentro de cada uma das populações de referência, foi comparada pelo teste qui-quadrado $\left(\chi^{2}\right)$, e utilizou-se a correlação de Pearson, a $5 \%$ de probabilidade, para avaliar as distribuições de frequência entre as populações CCM, CCS e CCMS.

O teste qui-quadrado e a análise de correlação foram realizadas no software SPSS 15.0 (SPSS, 2006), e as demais foram realizadas em planilha eletrônica.

\section{Resultados e Discussão}

As médias dos teores foliares e relações bivariadas entre nutrientes, obtidas para cada uma das populações de referência (Tabela 1), foram, em sua maioria, estatisticamente semelhantes (Tabela 2). Somente os teores de $\mathrm{Mg}$ e $\mathrm{Ca}$ nas folhas apresentaram diferenças significativas quando as normas DRIS foram comparadas entre si.

O desequilíbrio na nutrição de cálcio pode ser resultante de um antagonismo entre $\mathrm{K}$ e $\mathrm{Ca}$, em que o excesso do primeiro resultaria na diminuição do segundo (Marenco \& Lopes, 2007). Ayres \& Alfaia (2007) observaram maiores teores de K em pomares cultivados em monocultivo em relação aos cultivados em sistemas agroflorestais. Esses resultados podem estar relacionados às diferenças significativas observadas entre os teores nas normas (Tabela 2).

Entre os micronutrientes, $\mathrm{Zn}$ e $\mathrm{Fe}$ apresentaram teores médios acima de suas faixas de suficiência, e $\mathrm{Mn}$ e $\mathrm{Cu}$, abaixo (Tabela 1). A faixa de suficiência desses nutrientes para o cupuaçu é de 14-26, 118-242, 38-278 e 3,0 $\mathrm{mg} \mathrm{kg}^{-1}$ para $\mathrm{Zn}, \mathrm{Fe}, \mathrm{Mn}$ e $\mathrm{Cu}$, respectivamente (Salvador et al., 1994; Costa, 2006).

Apesar de as concentrações médias de alguns nutrientes nas folhas de cupuaçuzeiros, expressas nas normas, não terem coincidido com os teores considerados adequados por Salvador et al. (1994), não houve eliminação de nenhuma amostra nessa condição, em razão do fato de que teores críticos e faixas de suficiência devem ser utilizados com segurança somente em condições similares às condições em que foram determinados. Nas condições não controladas deste estudo, a concentração do nutriente no tecido vegetal depende de inúmeros outros fatores, como épocas da realização da amostragem e outros processos que podem afetar a taxa de acúmulo de matéria seca no tecido vegetal (Jarrel \& Beverly, 1981).

As médias das relações bivariadas entre nutrientes apresentaram elevada concordância nas populações de referência, com $87 \%$ das relações coincidentes entre as normas específicas e a norma geral (Tabela 2). Comparando-se as normas específicas entre si, o grau de concordância estatística reduz-se para $64 \%$ das relações. Esse resultado está de acordo com o que se esperava para normas DRIS específicas: que não fossem apropriadas para a avaliação nutricional de lavouras cujas condições de crescimento sejam distintas daquelas às quais a população de referência foi submetida (Santos et al., 2004; Silva et al., 2005; Rocha et al., 2007; Santana et al., 2008). Está de acordo também com o que se espera para normas gerais: que apresentem elevada aplicabilidade (Beaufils, 1973; Walworth \& Sumner, 1987).

A divergência entre as relações bivariadas das normas específicas ocorreu com a maioria dos nutrientes (Tabela 2), o que reforça a indicação de que não devem ser usadas como normas universais (Silva et al., 2005; Partelli et al., 2006; Rocha et al., 2007). Entretanto, quando a norma DRIS geral foi comparada com as normas específicas (CCM e CCS), as maiores divergências ocorreram em relações bivariadas que envolveram os nutrientes $\mathrm{N}, \mathrm{P}$ e $\mathrm{Zn}$.

A correlação entre o IBNm obtido da norma geral (CCMS) e o de normas específicas apresentou coeficiente elevado e significativo $(p<0,01)$ de $86 \%$ para CCMS vs. CCM e de $99 \%$ para CCMS vs. CCM. Em contrapartida, a correlação entre os IBNm obtidos das normas específicas entre si (CCM vs. CCS) jamais foi superior a $80 \%$, embora significativa. Esse resultado corrobora o observado com as relações bivariadas no que se refere à menor aplicabilidade de normas específicas.

Partelli et al. (2006), ao comparar normas DRIS específicas, observaram que o diagnóstico produzido 
Tabela 1. Média, desvio padrão (DP) e coeficiente de variação (CV, \%) dos teores foliares e relações bivariadas entre nutrientes, para as normas DRIS obtidas a partir de todos os pomares de cupuaçuzeiro avaliados (CCMS) e para as obtidas a partir de pomares cultivados em sistemas agroflorestais (CCS) e em monocultivo (CCM), na Amazônia Sul-Ocidental.

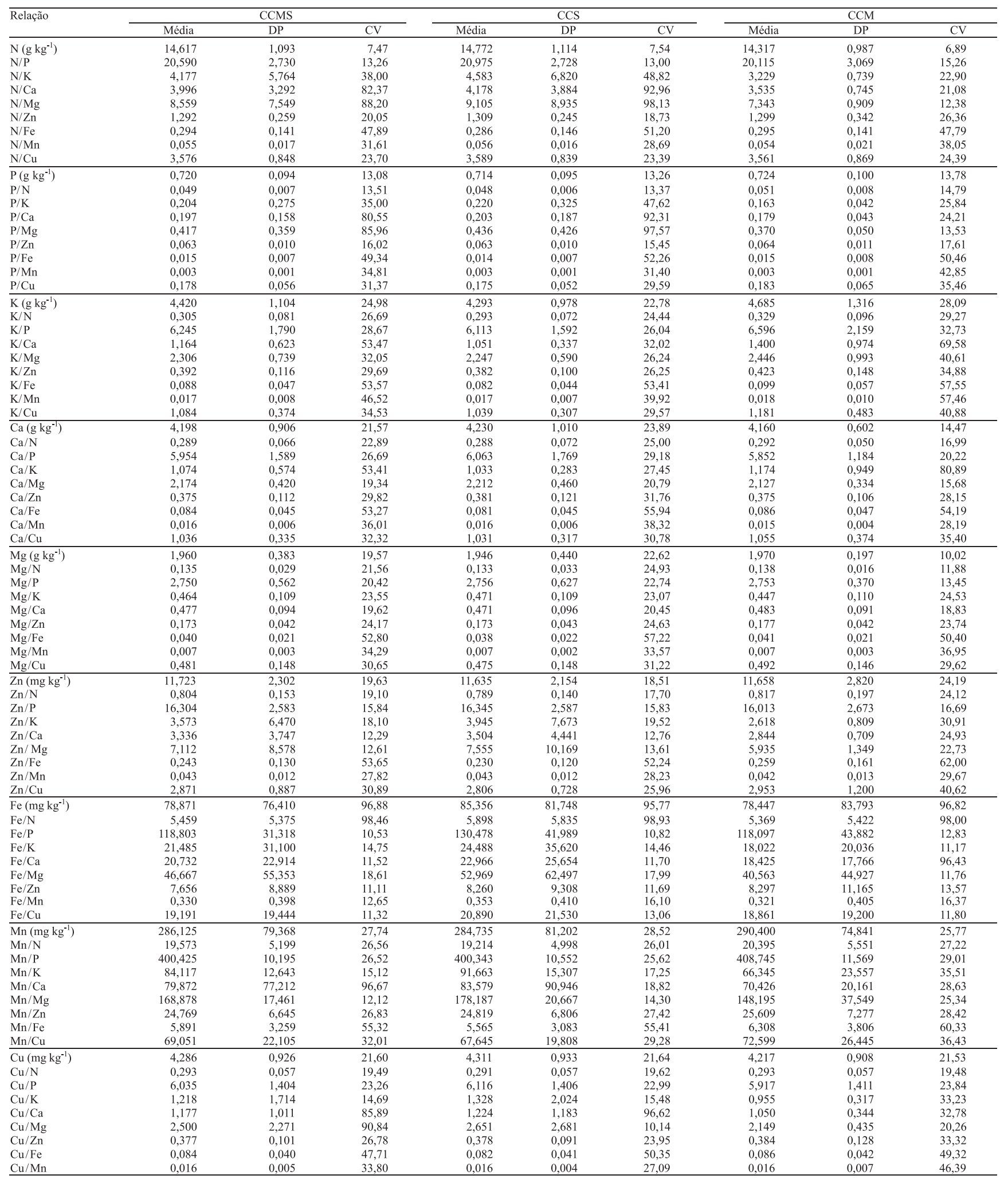

Pesq. agropec. bras., Brasília, v.45, n.1, p.64-71, jan. 2010 
Tabela 2. Comparação entre normas DRIS de pomares de cupuaçuzeiro cultivados em sistemas agroflorestais (CCS), em monocultivo (CCM) e com o uso de todos esses pomares indiscriminadamente (CCMS), na Amazônia Sul-Ocidental. ${ }^{(1)}$

\begin{tabular}{|c|c|c|c|}
\hline Relação & CCMS vs. CCS & CCMS vs. CCM & CCS vs. CCM \\
\hline $\mathrm{N}$ & $-0,63^{\mathrm{ns}}$ & $0,95^{\mathrm{ns}}$ & $1,36^{\mathrm{ns}}$ \\
\hline N/P & $-0,63^{\mathrm{ns}}$ & $0,57^{\text {ns }}$ & $0,98^{\mathrm{ns}}$ \\
\hline $\mathrm{N} / \mathrm{K}$ & $-0,29^{\mathrm{ns}}$ & $60,78^{*}$ & $85,09^{*}$ \\
\hline $\mathrm{N} / \mathrm{Ca}$ & $-0,23^{\mathrm{ns}}$ & $19,51^{*}$ & $27,16^{*}$ \\
\hline $\mathrm{N} / \mathrm{Mg}$ & $-0,30^{\text {ns }}$ & $68,92 *$ & $96,56^{*}$ \\
\hline $\mathrm{N} / \mathrm{Zn}$ & $-0,30^{\mathrm{ns}}$ & $-0,09^{\text {ns }}$ & $0,11^{\mathrm{ns}}$ \\
\hline $\mathrm{N} / \mathrm{Fe}$ & $0,23^{\text {ns }}$ & $-0,02^{\mathrm{ns}}$ & $-0,19^{\mathrm{ns}}$ \\
\hline N/Mn & $-0,13^{\text {ns }}$ & $0,26^{\mathrm{ns}}$ & $0,35^{\mathrm{ns}}$ \\
\hline $\mathrm{N} / \mathrm{Cu}$ & $-0,07^{\mathrm{ns}}$ & $0,06^{\mathrm{ns}}$ & $0,10^{\text {ns }}$ \\
\hline $\mathrm{P}$ & $0,27^{\mathrm{ns}}$ & $-0,13^{\text {ns }}$ & $-0,32^{\mathrm{ns}}$ \\
\hline $\mathrm{P} / \mathrm{N}$ & $0,63^{\text {ns }}$ & $-0,66^{\mathrm{ns}}$ & $-1,08^{\mathrm{ns}}$ \\
\hline $\mathrm{P} / \mathrm{K}$ & $-0,24^{\mathrm{ns}}$ & $42,41^{*}$ & $59,47 *$ \\
\hline $\mathrm{P} / \mathrm{Ca}$ & $-0,15^{\mathrm{ns}}$ & $13,42 *$ & $18,68^{*}$ \\
\hline $\mathrm{P} / \mathrm{Mg}$ & $-0,22^{\mathrm{ns}}$ & $51,51^{*}$ & $72,45^{*}$ \\
\hline $\mathrm{P} / \mathrm{Zn}$ & $0,10^{\mathrm{ns}}$ & $-0,43^{\text {ns }}$ & $-0,49^{\text {ns }}$ \\
\hline $\mathrm{P} / \mathrm{Fe}$ & $0,38^{\mathrm{ns}}$ & $-0,26^{\mathrm{ns}}$ & $-0,51^{\text {ns }}$ \\
\hline $\mathrm{P} / \mathrm{Mn}$ & $0,15^{\text {ns }}$ & $-0,05^{\text {ns }}$ & $-0,15^{\text {ns }}$ \\
\hline $\mathrm{P} / \mathrm{Cu}$ & $0,26^{\mathrm{ns}}$ & $-0,28^{\text {ns }}$ & $-0,46^{\text {ns }}$ \\
\hline $\mathrm{K}$ & $0,54^{\mathrm{ns}}$ & $-0,77^{\mathrm{ns}}$ & $-1,16^{\mathrm{ns}}$ \\
\hline $\mathrm{K} / \mathrm{N}$ & $0,68^{\text {ns }}$ & $-0,96^{\mathrm{ns}}$ & $-1,46^{\mathrm{ns}}$ \\
\hline $\mathrm{K} / \mathrm{P}$ & $0,34^{\mathrm{ns}}$ & $-0,63^{\text {ns }}$ & $-0,88^{\mathrm{ns}}$ \\
\hline $\mathrm{K} / \mathrm{Ca}$ & $3,42^{*}$ & $2,45^{*}$ & $8,38^{*}$ \\
\hline $\mathrm{K} / \mathrm{Mg}$ & $0,39^{\text {ns }}$ & $-0,58^{\mathrm{ns}}$ & $2,84 *$ \\
\hline $\mathrm{K} / \mathrm{Zn}$ & $0,39^{\mathrm{ns}}$ & $-0,85^{\text {ns }}$ & $2,16^{*}$ \\
\hline $\mathrm{K} / \mathrm{Fe}$ & $0,65^{\mathrm{ns}}$ & $-0,69^{\mathrm{ns}}$ & $-1,13^{\text {ns }}$ \\
\hline $\mathrm{K} / \mathrm{Mn}$ & $0,34^{\mathrm{ns}}$ & $-0,41^{\mathrm{ns}}$ & $2,49 *$ \\
\hline $\mathrm{K} / \mathrm{Cu}$ & $0,58^{\text {ns }}$ & $-0,82^{\mathrm{ns}}$ & $2,47 *$ \\
\hline $\mathrm{Ca}$ & $-0,15^{\mathrm{ns}}$ & $0,15^{\mathrm{ns}}$ & $2,82 *$ \\
\hline $\mathrm{Ca} / \mathrm{N}$ & $0,06^{\mathrm{ns}}$ & $-0,17^{\text {ns }}$ & $-0,19^{\text {ns }}$ \\
\hline $\mathrm{Ca} / \mathrm{P}$ & $-0,29^{\mathrm{ns}}$ & $0,23^{\mathrm{ns}}$ & $2,23 *$ \\
\hline $\mathrm{Ca} / \mathrm{K}$ & $4,10^{*}$ & $2,74 *$ & $11,22^{*}$ \\
\hline $\mathrm{Ca} / \mathrm{Mg}$ & $-0,38^{\text {ns }}$ & $0,40^{\text {ns }}$ & $0,64^{\mathrm{ns}}$ \\
\hline $\mathrm{Ca} / \mathrm{Zn}$ & $-0,25^{\mathrm{ns}}$ & $-0,02^{\mathrm{ns}}$ & $0,16^{\mathrm{ns}}$ \\
\hline $\mathrm{Ca} / \mathrm{Fe}$ & $0,28^{\text {ns }}$ & $-0,15^{\mathrm{ns}}$ & $-0,34^{\mathrm{ns}}$ \\
\hline $\mathrm{Ca} / \mathrm{Mn}$ & $-0,23^{\text {ns }}$ & $0,46^{\mathrm{ns}}$ & $0,59^{\text {ns }}$ \\
\hline $\mathrm{Ca} / \mathrm{Cu}$ & $0,07^{\mathrm{ns}}$ & $-0,19^{\mathrm{ns}}$ & $-0,23^{\text {ns }}$ \\
\hline $\mathrm{Mg}$ & $0,13^{\text {ns }}$ & $3,77^{*}$ & $4,98^{*}$ \\
\hline $\mathrm{Mg} / \mathrm{N}$ & $0,29^{\mathrm{ns}}$ & $3,15^{*}$ & $4,09 *$ \\
\hline $\mathrm{Mg} / \mathrm{P}$ & $-0,04^{\mathrm{ns}}$ & $-0,01^{\mathrm{ns}}$ & $2,87 *$ \\
\hline $\mathrm{Mg} / \mathrm{K}$ & $-0,27^{\mathrm{ns}}$ & $0,54^{\mathrm{ns}}$ & $0,71^{\mathrm{ns}}$ \\
\hline $\mathrm{Mg} / \mathrm{Ca}$ & $0,28^{\mathrm{ns}}$ & $-0,22^{\mathrm{ns}}$ & $-0,41^{\mathrm{ns}}$ \\
\hline $\mathrm{Mg} / \mathrm{Zn}$ & $0,04^{\mathrm{ns}}$ & $-0,35^{\mathrm{ns}}$ & $-0,36^{\text {ns }}$ \\
\hline $\mathrm{Mg} / \mathrm{Fe}$ & $0,30^{\mathrm{ns}}$ & $-0,16^{\mathrm{ns}}$ & $-0,36^{\mathrm{ns}}$ \\
\hline $\mathrm{Mg} / \mathrm{Mn}$ & $0,06^{\mathrm{ns}}$ & $0,05^{\mathrm{ns}}$ & $0,01^{\mathrm{ns}}$ \\
\hline $\mathrm{Mg} / \mathrm{Cu}$ & $0,20^{\mathrm{ns}}$ & $-0,25^{\mathrm{ns}}$ & $-0,38^{\text {ns }}$ \\
\hline $\mathrm{Zn}$ & $0,18^{\mathrm{ns}}$ & $0,09^{\text {ns }}$ & $-0,03^{\mathrm{ns}}$ \\
\hline $\mathrm{Zn} / \mathrm{N}$ & $0,45^{\mathrm{ns}}$ & $-0,28^{\text {ns }}$ & $-0,57^{\text {ns }}$ \\
\hline $\mathrm{Zn} / \mathrm{P}$ & $-0,07^{\mathrm{ns}}$ & $0,38^{\mathrm{ns}}$ & $0,41^{\mathrm{ns}}$ \\
\hline $\mathrm{Zn} / \mathrm{K}$ & $-0,24^{\mathrm{ns}}$ & $63,95^{*}$ & $89,93^{*}$ \\
\hline $\mathrm{Zn} / \mathrm{Ca}$ & $-0,18^{\mathrm{ns}}$ & $27,92 *$ & $39,24 *$ \\
\hline $\mathrm{Zn} / \mathrm{Mg}$ & $-0,21^{\mathrm{ns}}$ & $40,45^{*}$ & $56,85^{*}$ \\
\hline $\mathrm{Zn} / \mathrm{Fe}$ & $0,47^{\text {ns }}$ & $-0,39^{\mathrm{ns}}$ & $-0,71^{\mathrm{ns}}$ \\
\hline $\mathrm{Zn} / \mathrm{Mn}$ & $0,00^{\text {ns }}$ & $0,32^{\mathrm{ns}}$ & $0,30^{\text {ns }}$ \\
\hline $\mathrm{Zn} / \mathrm{Cu}$ & $0,35^{\text {ns }}$ & $-0,29^{\text {ns }}$ & $2,71^{*}$ \\
\hline $\mathrm{Fe}$ & $-0,37^{\text {ns }}$ & $0,02^{\text {ns }}$ & $0,27^{\mathrm{ns}}$ \\
\hline $\mathrm{Fe} / \mathrm{N}$ & $-0,35^{\mathrm{ns}}$ & $0,06^{\mathrm{ns}}$ & $0,30^{\text {ns }}$ \\
\hline $\mathrm{Fe} / \mathrm{P}$ & $-0,38^{\text {ns }}$ & $0,02^{\text {ns }}$ & $0,28^{\text {ns }}$ \\
\hline $\mathrm{Fe} / \mathrm{K}$ & $-0,40^{\mathrm{ns}}$ & $0,40^{\mathrm{ns}}$ & $3,16^{*}$ \\
\hline $\mathrm{Fe} / \mathrm{Ca}$ & $-0,41^{\mathrm{ns}}$ & $0,36^{\mathrm{ns}}$ & $0,62^{\text {ns }}$ \\
\hline $\mathrm{Fe} / \mathrm{Mg}$ & $-0,48^{\mathrm{ns}}$ & $0,39^{\text {ns }}$ & $0,69^{\text {ns }}$ \\
\hline $\mathrm{Fe} / \mathrm{Zn}$ & $-0,30^{\text {ns }}$ & $-0,23^{\mathrm{ns}}$ & $-0,01^{\mathrm{ns}}$ \\
\hline $\mathrm{Fe} / \mathrm{Mn}$ & $-0,25^{\mathrm{ns}}$ & $0,08^{\text {ns }}$ & $0,25^{\text {ns }}$ \\
\hline $\mathrm{Fe} / \mathrm{Cu}$ & $-0,37^{\mathrm{ns}}$ & $0,06^{\mathrm{ns}}$ & $0,31^{\mathrm{ns}}$ \\
\hline $\mathrm{Mn}$ & $0,08^{\text {ns }}$ & $-0,18^{\text {ns }}$ & $-0,23^{\text {ns }}$ \\
\hline $\mathrm{Mn} / \mathrm{N}$ & $0,31^{\mathrm{ns}}$ & $-0,53^{\mathrm{ns}}$ & $-0,74^{\mathrm{ns}}$ \\
\hline $\mathrm{Mn} / \mathrm{P}$ & $0,01^{\mathrm{ns}}$ & $-0,26^{\mathrm{ns}}$ & $-0,25^{\mathrm{ns}}$ \\
\hline $\mathrm{Mn} / \mathrm{K}$ & $-0,24^{\mathrm{ns}}$ & $30,29 *$ & $42,35^{*}$ \\
\hline $\mathrm{Mn} / \mathrm{Ca}$ & $-0,20^{\text {ns }}$ & $14,67^{*}$ & $20,35^{*}$ \\
\hline $\mathrm{Mn} / \mathrm{Mg}$ & $-0,22^{\mathrm{ns}}$ & $21,10^{*}$ & $29,42 *$ \\
\hline $\mathrm{Mn} / \mathrm{Zn}$ & $-0,03^{\text {ns }}$ & $-0,42^{\mathrm{ns}}$ & $-0,37^{\mathrm{ns}}$ \\
\hline $\mathrm{Mn} / \mathrm{Fe}$ & $0,46^{\text {ns }}$ & $-0,41^{\mathrm{ns}}$ & $-0,72^{\mathrm{ns}}$ \\
\hline $\mathrm{Mn} / \mathrm{Cu}$ & $0,30^{\text {ns }}$ & $-0,52^{\mathrm{ns}}$ & $-0,73^{\mathrm{ns}}$ \\
\hline $\mathrm{Cu}$ & $-0,12^{\mathrm{ns}}$ & $0,25^{\mathrm{ns}}$ & $0,33^{\mathrm{ns}}$ \\
\hline $\mathrm{Cu} / \mathrm{N}$ & $0,10^{\mathrm{ns}}$ & $-0,05^{\mathrm{ns}}$ & $-0,12^{\mathrm{ns}}$ \\
\hline $\mathrm{Cu} / \mathrm{P}$ & $-0,26^{\mathrm{ns}}$ & $0,28^{\text {ns }}$ & $0,46^{\text {ns }}$ \\
\hline $\mathrm{Cu} / \mathrm{K}$ & $-0,26^{\mathrm{ns}}$ & $29,16^{*}$ & $40,68^{*}$ \\
\hline $\mathrm{Cu} / \mathrm{Ca}$ & $-0,19^{\text {ns }}$ & $8,63^{*}$ & $11,82 *$ \\
\hline $\mathrm{Cu} / \mathrm{Mg}$ & $-0,27^{\mathrm{ns}}$ & $27,23 *$ & $37,94 *$ \\
\hline $\mathrm{Cu} / \mathrm{Zn}$ & $-0,05^{\mathrm{ns}}$ & $-0,23^{\text {ns }}$ & $-0,19^{\text {ns }}$ \\
\hline $\mathrm{Cu} / \mathrm{Fe}$ & $0,29^{\text {ns }}$ & $-0,10^{\text {ns }}$ & $-0,30^{\text {ns }}$ \\
\hline $\mathrm{Cu} / \mathrm{Mn}$ & $0.04^{\mathrm{ns}}$ & $0,04^{\mathrm{ns}}$ & $2,92 *$ \\
\hline
\end{tabular}

(1)ns Não significativo. *Significativo pelo teste $\mathrm{t}$ de Student e pelo teste $\mathrm{F}$, a $5 \%$ probabilidade. por cada uma das normas DRIS foi diferente para cada situação avaliada.

Ao se comparar os diagnósticos obtidos com o potencial de resposta à adubação, observou-se concordância superior a $90 \%$ entre os diagnósticos produzidos pela norma geral e os obtidos pelas demais (Tabela 3). Entretanto, quando os diagnósticos obtidos a partir de normas específicas foram comparados entre si, a concordância foi inferior a 70\%. A comparação entre os diagnósticos obtidos a partir de diferentes normas ratifica o que foi obtido com a comparação das relações bivariadas e dos IBNm. Esse último resultado, no entanto, deve ser considerado como mais veemente que os primeiros.

A frequência de distribuição de classes de potencial de resposta para cada nutriente em função dos diagnósticos gerados por cada uma das normas DRIS também se mostrou semelhante quando os diagnósticos foram produzidos pela norma CCMS ou pelas normas específicas (Tabela 4). Embora esse tipo de abordagem não indique, necessariamente, se cada pomar apresenta ou não o mesmo diagnóstico (Silva et al., 2005), fica evidente que, também nesse caso, a semelhança entre a frequência de distribuição de classes de potencial de resposta para cada nutriente é consideravelmente menor quando se comparam os diagnósticos resultantes das normas específicas entre si, o que está de acordo com Partelli et al. (2006), que observaram baixa concordância entre os diagnósticos produzidos por normas específicas em cafeeiros cultivados nos sistemas convencional e orgânico. A maior proporção de diagnósticos coincidentes quando se usam normas DRIS mais genéricas também foi observada em plantios de eucalipto (Wadt et al., 1998). A menor capacidade

Tabela 3. Percentagem de concordância nos diagnósticos nutricionais (excesso, equilíbrio e deficiência), obtidos a partir de normas DRIS geradas com todos os pomares de cupuaçuzeiro avaliados (CCMS) e com pomares cultivados em sistemas agroflorestais (CCS) e em monocultivo (CCM), na Amazônia Sul-Ocidental.

\begin{tabular}{lcccc}
\hline Nutriente & CCMS vs. CCS & CCMS vs. CCM & CCS vs. CCM & Média \\
\hline $\mathrm{N}$ & 97,4 & 84,3 & 83,7 & 88,45 \\
$\mathrm{P}$ & 99,3 & 92,8 & 71,9 & 88,02 \\
$\mathrm{~K}$ & 91,5 & 81,0 & 48,4 & 73,64 \\
$\mathrm{Ca}$ & 98,0 & 89,5 & 64,1 & 83,88 \\
$\mathrm{Mg}$ & 97,4 & 81,7 & 62,1 & 80,39 \\
$\mathrm{Zn}$ & 96,7 & 90,8 & 73,2 & 86,93 \\
$\mathrm{Fe}$ & 98,0 & 83,7 & 71,9 & 84,53 \\
$\mathrm{Mn}$ & 99,3 & 91,5 & 62,1 & 84,31 \\
\hline Média & 97,2 & 86,9 & 67,2 & 83,77 \\
\hline
\end{tabular}


preditiva de normas DRIS para lavouras que não estejam adequadamente representadas na população de referência também foi constatada em cana-de-açúcar (Reis Júnior \& Monnerat, 2003).

A capacidade de normas DRIS genéricas gerarem diagnósticos coerentes com os produzidos por normas DRIS específicas é particularmente importante, pois indica que o desenvolvimento de um único banco de dados que apresente uma população de referência representativa das diversas condições de cultivo dos pomares de cupuaçuzeiros, na Amazônia, pode ser utilizado para avaliação do estado nutricional de pomares cultivados em diferentes condições de manejo.

Tabela 4. Frequência em que os pomares avaliados apresentaram potencial de resposta à adubação positivo $(\mathrm{P})$, positivo ou nulo (PZ), nulo $(\mathrm{Z})$, negativo ou nulo (NZ) e negativo (N), de acordo com a avaliação do estado nutricional de plantas de cupuaçuzeiro, pelo uso de normas DRIS geradas a partir de pomares cultivados em sistemas agroflorestais (CCS), em monocultivo (CCM) e com o uso de todos esses pomares indiscriminadamente (CCMS).

\begin{tabular}{|c|c|c|c|c|c|c|}
\hline \multirow[t]{2}{*}{ Normas DRIS } & \multicolumn{5}{|c|}{ Frequência } & \multirow[t]{2}{*}{ Qui-quadrado } \\
\hline & $\mathrm{P}$ & $\mathrm{PZ}$ & $\mathrm{Z}$ & $\mathrm{NZ}$ & $\mathrm{N}$ & \\
\hline & \multicolumn{6}{|c|}{ Nitrogênio } \\
\hline CCMS & 5 & 7 & 123 & 7 & 11 & $2,5^{\operatorname{ns}(2)}$ \\
\hline $\mathrm{CCM}$ & 6 & 4 & 107 & 20 & 16 & $9,2^{\mathrm{ns}(3)}$ \\
\hline \multirow[t]{2}{*}{$\mathrm{CCS}$} & 5 & 10 & 122 & 10 & 6 & $11,5^{*(4)}$ \\
\hline & \multicolumn{6}{|c|}{ Fósforo } \\
\hline CCMS & 9 & 11 & 113 & 8 & 12 & $0,5^{\mathrm{ns}(2)}$ \\
\hline CCM & 10 & 10 & 114 & 11 & 8 & $1,4^{\operatorname{ns}(3)}$ \\
\hline \multirow[t]{2}{*}{$\mathrm{CCS}$} & 8 & 11 & 114 & 10 & 10 & $0,5^{\operatorname{ns}(4)}$ \\
\hline & \multicolumn{6}{|c|}{ Potássio } \\
\hline CCMS & 3 & 9 & 94 & 20 & 27 & $2,9^{\mathrm{ns}(2)}$ \\
\hline CCM & 9 & 22 & 85 & 18 & 19 & $10,4^{*(3)}$ \\
\hline \multirow[t]{2}{*}{ CCS } & 2 & 8 & 85 & 19 & 39 & $17,9 * *(4)$ \\
\hline & \multicolumn{6}{|c|}{ Cálcio } \\
\hline CCMS & 11 & 14 & 106 & 14 & 8 & $0,3^{\mathrm{ns}(2)}$ \\
\hline $\mathrm{CCM}$ & 17 & 16 & 92 & 17 & 11 & $3,2^{\operatorname{ns}(3)}$ \\
\hline \multirow[t]{2}{*}{$\mathrm{CCS}$} & 11 & 14 & 109 & 12 & 7 & $4,6^{\mathrm{ns}(4)}$ \\
\hline & \multicolumn{6}{|c|}{ Magnésio } \\
\hline CCMS & 8 & 8 & 120 & 9 & 8 & $0,7^{\mathrm{ns}(2)}$ \\
\hline $\mathrm{CCM}$ & 19 & 19 & 94 & 9 & 12 & $12,9 *(3)$ \\
\hline \multirow[t]{2}{*}{$\mathrm{CCS}$} & 8 & 5 & 122 & 10 & 8 & $17,1 * *(4)$ \\
\hline & \multicolumn{6}{|c|}{ Zinco } \\
\hline CCMS & 18 & 20 & 97 & 9 & 9 & $0,3^{\operatorname{ns}(2)}$ \\
\hline $\mathrm{CCM}$ & 10 & 14 & 111 & 10 & 8 & $4,4^{\operatorname{ns}(3)}$ \\
\hline \multirow[t]{2}{*}{ CCS } & 17 & 18 & 100 & 8 & 10 & $3,3^{\operatorname{ns}(4)}$ \\
\hline & \multicolumn{6}{|c|}{ Ferro } \\
\hline CCMS & 39 & 33 & 51 & 5 & 25 & $0,1^{\mathrm{ns}(2)}$ \\
\hline $\mathrm{CCM}$ & 21 & 30 & 74 & 3 & 25 & $10,3^{*(3)}$ \\
\hline \multirow[t]{2}{*}{$\mathrm{CCS}$} & 41 & 32 & 52 & 5 & 23 & $10,9^{*(4)}$ \\
\hline & \multicolumn{6}{|c|}{ Manganês } \\
\hline CCMS & 35 & 23 & 43 & 14 & 38 & $0,1^{\mathrm{ns}(2)}$ \\
\hline CCM & 39 & 15 & 54 & 8 & 37 & $4,8^{\operatorname{ns}(3)}$ \\
\hline $\mathrm{CCS}$ & 36 & 22 & 44 & 15 & 36 & $4,6^{\operatorname{ns}(4)}$ \\
\hline
\end{tabular}

(1)ns Não significativo. *e **Significativo pelo teste de Qui-quadrado $\left(\chi^{2}\right)$, a 5 e $1 \%$ de probabilidade, respectivamente. ${ }^{(2)} \mathrm{CCMS}$ vs. CCS. ${ }^{(3)} \mathrm{CCM}$ vs. CCMS. ${ }^{(4)}$ CCS vs. CCM.

\section{Conclusões}

1. A utilização de normas DRIS genéricas apresenta desempenho similar ao proporcionado por normas DRIS específicas na avaliação do estado nutricional de pomares de cupuaçuzeiros cultivados em diferentes condições de manejo.

2. Normas DRIS específicas apresentam aplicabilidade restrita às condições ambientais e de manejo de pomares de cupuaçuzeiros em que sua população de referência é cultivada.

\section{Referências}

ALFAIA, S.S.; AYRES, M.I. da C. Efeito de doses de nitrogênio, fósforo e potássio em duas cultivares de cupuaçu, com e sem semente, na região da Amazônia Central. Revista Brasileira de Fruticultura, v.26, p.320-325, 2004.

AYRES, M.I. da C.; ALFAIA, S.S. Calagem e adubação potássica na produção do cupuaçuzeiro em sistemas agroflorestais da Amazônia Ocidental. Pesquisa Agropecuária Brasileira, v.42, p.957-963, 2007.

BEAUFILS, E.R. Diagnosis and recommendation integrated system (DRIS): a general scheme for experimentation and calibration based on principles developed from research in plant nutrition. Pietermararitzburg: University of Natal, 1973. 132p. (Soil science bulletin, 1).

CANTERELLA, H. Nitrogênio. In: NOVAIS, R.F.; ALVAREZ V., V.H.; BARROS, N.F. de; FONTES, R.L.F.; CANTARUTTI, R.B.; NEVES, J.C.L. (Ed.). Fertilidade do solo. Viçosa: Sociedade Brasileira de Ciência do Solo, 2007. p.375-470.

CARMO, C.A.F. de S. do; ARAÚJO, W.S. de; BERNARDI, A.C. de C.; SALDANHA, M.F.C. Métodos de análise de tecidos vegetais utilizados na Embrapa Solos. Rio de Janeiro: Embrapa Solos, 2000. 41p.

COSTA, E.L. da. Exportação de nutrientes em frutos de cupuaçu (Theobroma grandiflorum) em três solos da Amazônia Central. 2006. 39p. Dissertação (Mestrado) -Universidade Federal do Amazonas, Manaus.

GALÍNDEZ, A.A.S.; CRUZ, J.G.; ZÉREGA, L.; RODRÍGUEZ, O.A.R.; OLIVEIRA, S.A. de; PÉREZ, V. de J.R. Normas preliminares DRIS desarrolladas para caña de azúcar a partir de un bajo número de muestras. Pesquisa Agropecuária Brasileira, v.44, p.1700-1706, 2009.

JARREL, W.M.; BEVERLY, R.B. The diluition effect in plant nutrition studies. Advances in Agronomy, v.34, p.197-224, 1981.

JONES, W.W. Proposed modifications of the diagnosis and recommendation integrated system (DRIS) for interpreting plant analyses. Communications in Soil Science and Plant Analysis, v.12, p.785-794, 1981.

LOPES, C.M.D'A.; SILVA, N.M. Impacto econômico da broca do cupuaçu, Conotrachelus humeropictus Field (Coleoptera: 
Curculionidae) nos estados do Amazonas e Rondônia. Sociedade Entomológica do Brasil, v.27, p.45-49, 1998.

MARENCO, R.A.; LOPES, N.F. Fisiologia vegetal: fotossíntese, respiração, relações hídricas e nutrição mineral. Viçosa: UFV, 2007. 469p.

MOURÃO FILHO, F. de A.A.; AZEVEDO, J.C.; NICK, J.A. Funções e ordem da razão dos nutrientes no estabelecimento de normas DRIS em laranja "Valência". Pesquisa Agropecuária Brasileira, v.37, p.185-192, 2002.

PARTELLI, F.L.; VIEIRA, H.D.; MONNERAT, P.H.; VIANA, A.P. Estabelecimento de normas DRIS em cafeeiro conilon orgânico e convencional no Estado do Espírito Santo. Revista Brasileira de Ciência do Solo, v.30, p.443-451, 2006.

PRADO, R. de M.; ROZANE, D.E.; VALE, D.W. do; CORREIA, M.A.R.; SOUZA, H.A. de. Nutrição de plantas: diagnose foliar em grandes culturas. Jaboticabal: Unesp, 2008. 301p.

REIS JUNIOR, R. dos A. Dris norms universality in the corn crop. Communications in Soil Science and Plant Analysis, v.33, p.711-735, 2002.

REIS JÚNIOR, R. dos A.; MONNERAT, P.H. Norms establishment of the diagnosis and recommendation integrated system (DRIS) for nutritional diagnosis of sugarcane. Pesquisa Agropecuária Brasileira, v.38, p.277-282, 2003.

ROCHA, A.C. da; LEANDRO, W.M.; ROCHA, A.O.; SANTANA, J. das G.; ANDRADE, J.W. de S. Normas DRIS para cultura do milho semeado em espaçamento reduzido na região de Hidrolândia, GO, Brasil. Bioscience Journal, v.23, p.50-60, 2007.

SALVADOR, J.O.; MURAOKA, T.; ROSSETO, R.; RIBEIRO, G. de A. Sintomas de deficiências nutricionais em cupuaçuzeiro (Theobroma grandiflorum) cultivado em solução nutritiva. Scientia Agricola, v.51, p.407-414, 1994.

SANTANA, J. das G.; LEANDRO, W.M.; NAVES, R.V.; CUNHA, P.P. da. Normas DRIS para interpretação de análises de folha e solo, em laranjeira pêra, na região central de Goiás. Pesquisa Agropecuária Tropical, v.38, p.109-117, 2008.

SANTOS, A.L. dos; MONNERAT, P.H.; CARVALHO, A.J.C. de. Estabelecimento de normas DRIS para o diagnóstico nutricional do coqueiro-anão verde na região norte fluminense. Revista Brasileira de Fruticultura, v.26, p. 330-334, 2004.

SILVA, G.G.C. da; NEVES, J.C.L.; ALVAREZ V., V.H.; LEITE, F.P. Avaliação da universalidade das normas DRIS, M-DRIS e CND. Revista Brasileira de Ciência do Solo, v.29, p.755-761, 2005.

SPSS. SPSS Graduate Pack. Version 15.0. Chicago: SPSS, 2006.

DRIS. Sistema integrado de diagnose e recomendação. Disponível em: <http://www.dris.com.br/>. Acesso em: 05 mar. 2010.

VELOSO, C.A. da C.; PEREIRA, W.L.M.; CARVALHO, E.J.M. Diagnose nutricional pela análise foliar de pomares de laranjeiras no nordeste paraense. Revista de Ciências Agrárias, n.38, p.47-55, 2002.

WADT, P.G.S. Relationships between soil class and nutritional status of coffee plantations. Revista Brasileira de Ciência do Solo, v.29, p.227-234, 2005.

WADT, P.G.S.; NOVAIS, R.F. de. O monitoramento nutricional frente aos métodos diagnósticos no planejamento das adubações. In: SIMPÓSIO SOBRE MONITORAMENTO NUTRICIONAL PARAA RECOMENDAÇÃO DAADUBAÇÃO DE CULTURAS, 1999, Piracicaba. Monitoramento nutricional para a recomendação de adubação de culturas: anais. Piracicaba: Potafos, 1999. p.70-80.

WADT, P.G.S.; NOVAIS, R.F. de; ALVAREZ V., V.H.; FONSECA, S.; BARROS, N.F. Valores de referência para macronutrientes em eucalipto obtidos pelos métodos DRIS e chance matemática. Revista Brasileira de Ciência do Solo, v.22, p.685-692, 1998.

WALWORTH, J.L.; SUMNER, M.E. The diagnosis and recommendation integrated system (DRIS). Advances in Soil Sciences, v.6, p.149-188, 1987.

$\overline{\text { Recebido em } 29 \text { de junho de } 2009 \text { e aprovado em } 28 \text { de dezembro de } 2009}$ 